\section{Ueber das pathologisch-anatomische Bild der „Kriegsnephritis“.}

Von Stabsarzt Prof. Dr. Gotthold Herxheimer,

z. Z. bei einer Kriegslazarettabteilung.

(ScliluB aus Nr. 31.)

Fragen wir nun, in welche der drei Gruppen unsere „Kriegsnephritis“" gehört, so ist die Frage leicht zu beantworten. Ausnahmslos liegen die Hauptveränderungen in den Glomeruli. Da diese zum allergrößten Teil ergriffen sind, liegt auch eine diffuse Erkrankung vor, und zwar eine zu der zweiten, eben gekennzeichneten Klasse gehörende, also zu der Glomerulonephritis, und da wieder, wie die erst beginnenden Fälle zeigen, im Anfang weuigstens zu der dritten obeu aufgestellten Form, d. h. der möglichst reinen Glomerulitis. Fr. Pick ${ }^{1}$ ) vermutet auf Grund klinischer Beobachtungen mit Recht, daß es sich um eine vaskuläre Nierenschädigung handelt, irrt aber, wenn er eine herdweise, nicht diffuse Nephritis annimmt, während Benda ${ }^{2}$ ) aus einem älteren Fall mit Recht auf eine Nephritis vom glomerulären Typus schließt. Ja, es kann an der Hand der Frühstadien gesagt werden, daß so reine Formen von Glomerulitis sonst geradezu selten zu Gesicht kommen. Hier liegt also, wie eben gekennzeichnet, der letzte Angriffspunkt in den Kapillaren der Glomeruli. Sicher schließt sich ja der allgemeinen Auffassung nach an diesen Angriffspunkt zumeist echte Entzündung mit Exsudatbildung an, ja es ist fast die Nephritis katexochen nach der heutigen Auffassung, aber die ganzeu beginnenden Fälle zeigen einerseits von der für die Eutzündung charakteristischeu Exsudatbildung noch kaum etwas, anderseits eine offenbar auf die Kapillarendothelschädigung folgende Hyperregeneration derselben als das auffälligste morphologisch wahrnehmbare Kennzeichen, während bei einer gewöhnlichen „Entzündung" die Schädigung der Gefäße morphologisch in Veränderungen an diesen nicht erkennbar zu sein pflegt. Ich glaube daher, daß die erste Glomerulusveränderung an sich keine Entzündung bedeutet, nur daß sich an sie in den schweren Fällen, bzw. wenn die Noxe von vornherein stärker einwirkt, eine solche anschließt. Dieser Gesichtspunkt ist aber, wie mir scheint, wichtig, denn wenn wir bedenken, daß selbst die beginnenden Fälle, die zur Sektion führen, schon ausnahmsweise schwere sind die allermeisten Fälle verlaufen ja leicht und schnell-, so dürfen wir für diese wohl eine noch geringere Veräuderung der Glomeruli, d.h. eine reine Kapillarwandschädigung annehmen. Anatomisch ist nicht zu entscheideu, ob diese nicht Teilerscheinung einer allgemeinen Kapillarschädigung - auch peripherischer Kapillaren - ist; morphologisch fand sich bei darauf gerichteten Untersuchungen nichts dafür Sprechendes, doch ist das gut annehmbar und wird von zahlreichen Seiten klinisch angenommen. Verlaufen die Fälle aber schwer und führen doch erst nach einigen Wochen zum Tode, so finden wir weit schwerere Veränderungen, d. h. das subakute bzw. subchronische Stadium der Glomerulonephritis mit qusgeprägtesten Kapselepithelwucherungen, sogenannten „Halbmonden" - Fahr nennt diese Form die extrakapillare der subakuten Glomerulonephritis - und schwerster Schädigung des Parenchyms.

1) Verein deutscher Aerzte in Prag 17. XII. 1916. Siehe M. m. W.

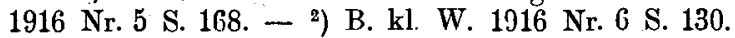

Durch die verschiedenen anatomischen Bilder und diese ganze Auffassung wird das so sehr variable klinische Bild der ,Kriegsnephritis" ebensogut erklärt wie einzelne Symptome: die Herabsetzung der Urinmenge eventuell bis zur Anurie, die Fiweißmenge des Urins, das vor allem auch von Pick als besonders auffallend hervorgehobene hartnäckige Auftreten roter Blutkörperchen und Zylinder im Harn, zugleich aber auch das Vermissen dieser morphologischen Elemente im Urin - besonders der Zylinder - in zahlreichen anderen Fällen mit viel Fiweiß. Die nach den Untersuchungen von Nonnenbruch im Gegensatz zu anderen Beobachtern häufig vorhandene Blutdrucksteigerung wird sowieso jetzt zumeist auf Veränderungen der Nierengefäße bezogen - geringere, wie in den vorliegenden Fällen, auf solche der Glomeruluskapillaren (Glomerulonephritis), die höchsten Grade auf die atherosklerotischen Gefäßveränderungen (sogenannte genuine Schrumpfniere, s. oben). Endlich die Oedeme, zumeist das Hauptsymptom der "Kriegsnephritis", werden ja jetzt klinischerseits - ich verweise z. B. auf die neueste Abhandlung von Hessi') - zumeist, gestiutzt auf Beobachtungen und Tierversuche, als Folge einer Srhädigung der Kapillarwandzellen, insbesondere der Nieren, aufgefaßt. Für die eventuell vorhandene Urämie a ber ist die letzte Erklärung ja überhaupt noch umstritten.

Stellt nach alledem die ,Kriegsnephritis" keine neue Krankheit sui generis dar, sondern liegt eine Glomerulitis bzw. Glomerulonephritis vor, welche alle Bilder von einfachen (Glomerulus-)Kapillarschädigungen und -veränderungen bis zur schwersten Glomerulonephritis umfaßt, so scheint mir diese anatomische Klärung und Auffassung des Leidens nicht nur das klinische Bild gut zu erklären, sondern auch wichtige ätiologische Anhaltspunkte zu geben. Allgemein ist zu bemerken, daß Gefäßschädigungen mehr, als früher angenommen, auf Infektionen zu beziehen sind, wie Wiesel, Wiesner u. a. nachwiesen. Speziell die Glomerulonephritis aber, als welche sich unsere Kriegsnephritis darstellt, wird von allen Seiten als infektiös en Ursprungs anerkannt. Am häufigsten ist sie bekanntlich im Anschluß an Scharlach und soll es früher mehr als jetzt gewesen sein. Dabei haben besonders Aschoff und Fahr-Volhard darauf hingewiesen, daß es hier besonders Streptokokken sind, welche ursächlich in Betracht kommen. Denn sie betonen, daß auch bei den anderen Krankheiten, bei denen sich Glomerulonephritis findet, Streptokokken als schädigendes Agens anzunehmen sind - so bei Anginen, Gelenkrheumatismus, Erysipel usw. Auch Löhlein betonte schon, daß in seinen Fällen, welche er in früheren Stadien genau untersuchen konnte, stets eine Strektokokkeninfektion sicher vorlag oder - anamnestisch usw. - zu erschließen war. Daneben kommen nach Nauwerck, Aschoff, Fahr-Volhard eventuell noch Pneumokokken in Betracht. (Für Tuberkulose wird die Frage offengelassen - eventuell käme auch hier Mischinfektion mit Kokken in Betracht - , ich habe bei eingehenden Untersuchungen von Nieren Tuberkulöser früher nichts dergleichen gesehen; Tuberkulose ist ja in unseren Fällen - bis auf zwei - auch ätiologisch auszuschließen.) Hiernach ist a priori mit großer Wahrscheinlichkeit anzunehmen, daß auch bei der "Kriegsnephritis" eine Infektion vorliegt. Der geringe, akute, weiche Milztumor, den wir in allen unseren Fällen fanden - eventuell zu gering, um klinisch nachweisbar zu sein —, weist auch direkt darauf hin; in zwei Fällen eventuell auf die Lungen-Pleura-Komplikation zu beziehen, war er doch sonst Teilerscheinung der Erkrankung selbst. In demselben Sinne ist das anfänglich fast stets vorhandene Fieber, sind allgemeine Symptome, wie Kopfschmerzen, Mattigkeit usw., zu deuten. Auch die wenigen Fälle von Erkrankung von Pflegepersonal sprechen in demselben Sinne. Nach dem oben Dargelegten ist aber eine Streptokokkeninfektion das Wahrscheinlichste. Interessant und als Hinweis hierauf sind auch unsere Strepto- bzw. Diplokokkenbefunde in Fall 9 und 10 (s. dort) zu verwerten; allerdings nur in dem allgemeinen Sinne, daß in diesen Fällen die Kokken im Körper vorhanden waren (Empyem im Anschluß an Bronchial-Lungenveränderungen). Von Interesse ist besonders auch die Eing angs pforte. Nach Volhards Zusammenstellung ist für drei Viertel aller Nephritiden bekannter Aetiologie die Mandel bzw. der lymphatische Rachenring als solche anzunehmen. In einem unserer 
Fälle bestand auch eine Angina, in den anderen aber, wie auch sonst fast stets, nicht. Das beweist natürlich an sich noch nichts, zumal an der Eingangspforte eine Erkrankung nicht morphologisch wahrnehmbar zu sein braucht. Nun findet sich aber häufig klinisch als Initialsymptom eine andere Erkrankung der oberen Respirationswege, nämlich eine akute Bronchitis, und auch anatomisch war in unseren Fällen eine schwere Bronchitis (und Tracheitis) ausnahmslos, eventuell auch eine Laryngitis, nachweisbar. Auch Bronchopneumonien schlossen sich öfters als schwere Komplikation an. Derartige Affektionen sind nun sehr häufig auf Streptokokken zurückzuführen, und da liegt es für unsere Fälle sehr nahe, in dieser die Erscheinungen einleitenden Erkrankung der oberen Luftwege die Eingangspforte auch für die Nierenerkrankung zu sehen. Weit unwahrscheinlicher als Streptokokken wären hier Pneumokokken. Als direkte Noxe für die Niere kommen dann wohl nicht die. Kokken selbst, sondern nach allgemeinen Anschauungen, die GefäBschädigungen und besonders die Glomerulonephritis betreffend, im wesentlichen ihre Toxine in Betracht. Kokken habe ich auch in den Schnitten der Nieren niemals (im Fall 10 lagen offenbar erst ganz zuletzt erfolgte Kokkenembolien vor) gefunden, und so wäre es auch ohne weiteres erklärt, warum bei bakteriologischen Untersuchungen von Kriegsnephritikern im Blute - und ebenso in der Milz unseres ersten Falles, wo darauf untersucht wurde - Bakterien nicht nach. gewiesen werden konnten.

Auch an das Bacterium coli und den Darm als Eingangspforte ist gedacht worden, so von Klein und $\mathrm{Pula} \mathbf{y}^{1}$ ), da sie aus dem Harn in 15 Fällen Bacterium coli züchten konnten und ihre Kranken an Dysenterie, Typhus, Enteritis zuvor gelitten hatten. Aber eine solche Annahme ist mir sehr viel unwahrscheinlicher. Das erste Argument von Klein-Pulay ist nicht hinreichend beweisend, und das zweite konnte sich anamnestisch bei zahlreichen anderen Patienten - im Westen nicht nachweisen lassen. Anatomisch haben wir einige Male eine geringe Enteritis - wie sie sich auch sonst häufig findet - gesehen, zumeist aber völlig vermißt; das beweist natürlich auch noch nichts dagegen, $\mathrm{da} B$ eine Enteritis vorangegangen. Aber gegen diese Auffassung scheint mir entschieden zu sprechen, daB einerseits Glomerulonephritis bei Bacterium coli-lnfektion früher nie sicher nachgewiesen wurde - im Gegensatz zu der so häufigen Streptokokkeninfektion - und daß anderseits gerade die eitrigen Entzïndungen von Blase und Nierenbecken-Nieren so ganz gewöhnlich durch das Bacterium coli hervorgerufen werden, hier aber nie von solchen die Rede ist. Auch in der dem Vortrag von Klein-Pulay folgenden Besprechung in der Wiener Gesellschaft der Aerzte lehnten die meisten Redner die ätiologische Bedeutung der vorausgegangenen Darmerkrankung ab. Pick fand auch keine Kolibakterien. Der Darm wurde auch in anderer Weise als Eingangspforte angenommen, indem von bakterieller Ursache abgesehen und ein mit der Nahrung zusammenhängender Faktor ätiologisch herangezogen wirde. Einmal dachte man an Stoffe, welche der Nahrung fehlten (Albu. Schlesinger); aber hiergegen, als grundlegendes ätiologisches, nicht eventuell als disponierendes Moment, spricht wohl schon die Form der anatomischen Läsion - die Kapillarschädigung und Entzündung -, und auch die Infektionsmilz ist damit kaum vereinbar. Anderseits dachte man an Schädigungen durch Stoffe der Nahrung. Aber auch dies ist bei den anatomisch festgestellten Veränderungen weit unwahrscheinlicher als eine (Streptokokken.) Infektion. Und ebenso spricht der Um. stand, daß dieselbe Erkrankung auch von englischer Seite mitgeteilt wurde, gegen eine solche Auffassung.

Ist somit eine Streptokokkeninfektion, mit den oberen Luftwegen als Eingangspforte, als ätiologische Grundlage der ,Kriegsnephritis" das Wahrschein. lichste, so handelt es sich hier wohl sicher um fast ubiquitäre, an sich wenig virulente Kokken, welche ihre pathogene Einwirkung erst unter ganz bestimmten Umständen entfalten können. Die besondere Dis. position spielt somit hier offenbar eine außerordentlich wichtige Rolle, noch mehr als wie sie heute im Gegensatz zur ersten rein bakteriologischen Aera bei Infektionen mit vollem Recht wieder allgemein betont wird. Als disponierendes Moment kommt nun erfahrungsgemäß und experimentell begründet bei Nephritiden und Gefäßschädigungen vor allem intensive Kälteeinwirkung und Durchnässung in Betracht.

1) Wiener Gesellschaft deutscher Aerzte 22. X. 1915. Siehe M. m. W. 1915 Nr. 4 S. 1616.
Nach allem scheint es aber, daß diese Faktoren nicht an sich - wie früher vielfach angenommen - die Nephritis setzen, sondern da B sie nur die bakterielle Infektion begünstigen, wobei Bouchard, Pasteur, Wagner, Loele, Filehne, Kisskalt die Erkältung der Haut als besonders günstigen Nährboden für schon im Körper befindliche Bakterien betrachten, Chodomski, Lipari, Seidel hingegen annehmen, daB die Bakterien unter solchen Bedingungen virulenter werden (zitiert nach $B\left(\mathrm{um}^{1}\right.$ ). Luedke $\mathrm{e}^{2}$ ) fand auch bei Erkältungsnephritiden Streptokokken im Urin.

Bei der Kriegsnephritis wird nun, wie es scheint, eine derartige Erkältung und vor allem Durchnässung in der Tat anamnestisch besonders häufig angeschuldigt, sie könnte zugleich die Disposition zur Bronchitis darstellen.

Auch Bl u r hat die Bedeutung der Erkältung bei der Kriegsnephritis schon betont, ähnlich $\mathrm{Moss} \mathrm{e}^{8}$ ), während $\mathrm{Knack}^{4}$ ) sie mehr ablehnt Pick hat auch schon auf die Wichtigkeit der Kombination von Infektion und Durehnässung bei ihr hingewiesen. Interessant ist auch, daß, wie es scheint, gerade in Gebieten, wo infolge der Erdbeschaffenheit Durchnässung am seltensten, Mangel an Licht, der sicher ganz zu Unrecht auch ätiologisch beschuldigt wurde, aber relativ sehr groß ist, die „Kriegsnephritis“ am wenigsten beobachtet $\mathrm{zu}$ sein scheint.

In anderen Fällen kommen andere disponierende Momente in Betracht. So hat Blum auch der individuellen Prädisposi. tion mit Recht einen weiten Spielraum eingeräumt, insbesondere einer angeborenen oder erworbenen Minderwertigkeit des Gefäßsystems und somit Störung der Durch. blutung der Niere. In diesem Sinne sind wohl die Fälle von Kriegsnephritis zu deuten, welche bei Bleiarbeitern - wie auch in einem unserer Fälle -, alten Nephritikern und dergleichen auftreten. Auch in unserem Falle mit Hypoplasie der einen, Hypertrophie der anderen Niere ist wohl eine Organdisposition zur Nierenerkrankung anzuerkennen. Auch die Tuberkulose wie in zweien unserer Fälle - kann in diesem Sinne herangezogen werden. Bei individueller Minderwertigkeit der Nierendurchblutung mögen auch sehr langes Stehen und Ermüdung zur Erkrankung disponieren. In diesem Zusammenhange ist es von Interesse, daß auch nach Abheilung der „Kriegsnephritis", wie mir berichtet wurde, noch eine Art orthostatische Albuminurie einige Zeit zurückbleiben kann, die auch als besondere Disposition der Kapillargefäße der Niere zur Durchlässigkeit für Eiweiß aufzufassen ist, hier als Ueberbleibsel der von uns als Grundlage der Krankheit angenommenen Kapillarschädigung anzusehen. Alle genannten, im Organismus gelegenen und von außen einwirkenden dispositionellen Mo. mente können konkurrieren, um den sonst harm. loseren Bakterien den Angriff $z u$ erleichtern.

Was endlich den Ausgang der Krankheit betrifft, so scheint es sichergestellt, daß die allermeisten akuten Glomerulonephritiden ausheilen können - unser Fall 10 und eventuell 11 sind auch in dieser Richtung interessant; bei der mehr reinen Kapillarschädigung, wie sie für die leichten Fälle von „Kriegsnephritis" anzunehmen ist, ist dies wohl erst recht der Fall. Anderseits werden die Fälle, die nicht abheilen und auch nicht in frühen Stadien tödlich enden, zu dem Bilde der Schrumpfniere mit allen ihren Symptomen führen. Wenn bisher gerade bei der Glomerulonephritis die anatomischen Bilder der Ausheilung einerseits, des direkten Zusammenhanges der Anfangs- und Endbilder des Prozesses anderseits schwer $\mathrm{zu}$ verfolgen waren, so wird jetzt bzw. später bei Leuten, die an "Kriegsnephritis" gelitten, die wir als anatomisch gleichbedeutend mit Glomerulitis bzw. Glomerulonephritis setzen dürfen, zu Studien nach beiden Richtungen hin Gelegenheit gegeben sein. Zunächst aber scheint mir die anatomische Verfolgung dieser jetzt so wichtigen Erkrankung ihr Bild geklärt und somit Schlüsse auf Aetiologie, Verlauf und Werdegang der Erkrankung an die Hand gegeben zu haben.

Zusammenfassung. Die „Kriegsnephritis" ist eine Nierenund besonders Glomeruluserkrankung von einfacher (Glomerulus-)Kapillarschädigung bis zu schwerer Glomerulonephritis; ich habe bisher anatomisch das akute a id subakute Stadium beobachtet. Aetiologisch sind wahrscheinlich Toxine von (Strepto-)Kokken anzuschuldigen, die wohl durch die oberen

1) W. kl. W. 1915 Nr. 46. - ${ }^{2}$ ) Fortschr. d. deutschen Klinik 2 1911. - ${ }^{3}$ D. m. W. 1916 Nr. 3. $-{ }^{4}$ ) Aerztl. Verein ¡in Hamburg 11 I. 1916 . Siehe D. m. W. 1916 Nr. 18 S. 559 
Luftwege - Bronchitis, Tracheïtis, Laryngitis - aufgenommen werden, wobei disponierenden Momenten, in erster Linie Durchnässung und Erkältung, sodann auch individuellen Verhältnissen der Nierendurchblutung, eventaell auf Grund früherer Erkrankungen, große Bedeutung zukommt. 